\title{
Measurements of differential top quark pair production cross sections as a function of kinematic event variables at $13 \mathrm{TeV}$ at CMS
}

\section{Burns* on behalf of the CMS Collaboration}

Department of Physics, University of Bristol

IIHE, Vrije Universiteit Brussel

E-mail: Douglas.Burnsebristol.ac.uk

\begin{abstract}
Measurements of differential top quark pair production cross sections are presented in the singlelepton decay channel, as a function of a number of kinematic event variables. The measurements are performed with proton-proton collision data collected by the CMS experiment at the LHC during 2016 at $\sqrt{s}=13 \mathrm{TeV}$, with an integrated luminosity of $35.9 \mathrm{fb}^{-1}$. The data are compared to a variety of state-of-the-art leading-order and next-to-leading-order top quark pair production simulations.
\end{abstract}

ICHEP 2018, International Conference on High-Energy Physics

4th-8th July 2018

Seoul, Korea

${ }^{*}$ Speaker. 
This note presents a differential top quark pair production (tit) cross section paper produced by CMS [1] using the 2016 data set [2]. Both normalized and absolute differential cross sections are measured in the single lepton decay channel to particle level in a visible phase space. The measurements are performed as a function of several kinematic event variables (which do not require $t \bar{t}$ system to be reconstructed), namely: the jet multiplicity $N_{\text {jets }}$, the magnitudes of the signal lepton transverse momentum and pseudorapidity $p_{\mathrm{T}}^{\ell}$ and $\left|\eta^{\ell}\right|$, the sum of the hadronic activity of the event $H_{\mathrm{T}}$, the total activity of the event $S_{\mathrm{T}}$, the missing transverse momentum $p_{\mathrm{T}}^{\text {miss }}$, and the transverse momentum of the leptonically decaying $\mathrm{W}$ boson $p_{\mathrm{T}}^{\mathrm{W}}$. Full comparisons of the differential cross sections are performed for several state-of-the-art $\mathrm{tt}$ generators at leading order (LO) and next-to-leading order (NLO), including $\chi^{2}$ goodness-of-fit tests.

Figure 1 shows the differential cross section with respect to $N_{\text {jets }}$ has good agreement with the NLO PowheG+Pythia model. This is reinforced by the outcome of the $\chi^{2}$ goodness-of-fit test with respect to the PowhEG+PythiA model shown in Tab. 1. The underlying event tune used in the model is derived using 7 and $8 \mathrm{TeV}$ measurements with respect to the jet multiplicity and this measurement confirms the tune continues to accurately describe the jet multiplicity on a larger data set with higher centre-of-mass energy. The differential cross sections with respect to event variables correlated to the top quark $p_{\mathrm{T}}$ display a trend of being softer in data than in simulation, which is more pronounced for LO models than NLO models. The differential cross section with respect to $\left|\eta^{\ell}\right|$ performs poorly in the goodness-of-fit test for all tested models. The NLO generators are concluded to be generally consistent with the data, although no one generator describes the data perfectly and the LO generator does not describe the data. Any differences seen with the Powheg+Pythia model are covered by the inclusion of the theoretical uncertainties within the model to the goodness-of-fit tests, also shown in Tab. 1. At this point the measurements are limited by the systematic uncertainties, dominated for both the normalized and absolute cross section by the uncertainty in the jet energy scale. Other large uncertainties are primarily related to the modelling of the parton shower.

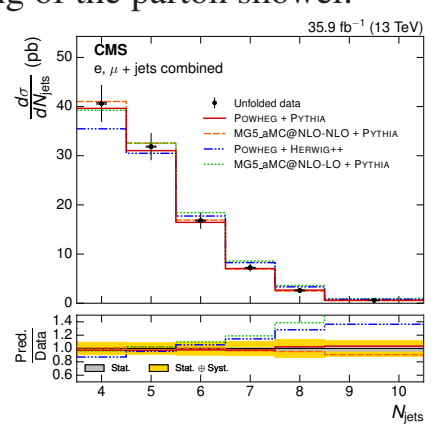

Figure 1: The absolute differential cross section measurement with respect to $N_{\text {jets }}$ [2].

\begin{tabular}{c|cc|cc} 
& \multicolumn{2}{|c|}{ PowhEG+PyTHIA } & With MC theoretical uncertainties \\
& $\chi^{2} /$ ndf & $p$-value & $\chi^{2}$ /ndf & $p$-value \\
\hline$N_{\text {jets }}$ & $2.2 / 6$ & 0.90 & $1.7 / 6$ & 0.95 \\
$H_{\mathrm{T}}$ & $23 / 13$ & 0.05 & $4.3 / 13$ & 0.99 \\
$S_{\mathrm{T}}$ & $19 / 13$ & 0.11 & $4.7 / 13$ & 0.98 \\
$p_{\mathrm{T}}^{\mathrm{miss}}$ & $13 / 6$ & 0.05 & $3.1 / 6$ & 0.80 \\
$p_{\mathrm{T}}^{\mathrm{W}}$ & $17 / 7$ & 0.02 & $2.7 / 7$ & 0.91 \\
$p_{\mathrm{T}}^{\ell}$ & $20 / 17$ & 0.28 & $14 / 17$ & 0.68 \\
$\left|\eta^{\ell}\right|$ & $16 / 8$ & 0.04 & $15 / 8$ & 0.06
\end{tabular}

Table 1: Results of goodness-of-fit tests of the absolute cross sections in data and the Powheg+Pythia model.

\section{References}

[1] CMS Collaboration, The CMS Experiment at the CERN LHC, JINST 3 (2008) S08004.

[2] CMS Collaboration, Measurements of differential cross sections of top quark pair production as a function of kinematic event variables in proton-proton collisions at $\sqrt{s}=13 \mathrm{TeV}$, JHEP 06 (2018) 002 [1803.03991]. 